\title{
TITEL
}

Henning Rosenau/Igor Sorge

\section{Gewerbsmäßige Suizidförderung als strafwürdiges Unrecht? Kritische Anmerkungen zum Regierungsentwurf über die Strafbarkeit der gewerbsmäßigen Förderung der Selbsttötung $(\mathbb{2} 217 \mathrm{StGB}-\mathrm{E})$}

\section{Einleitung}

Im Jahr 2005 entstand der deutsche Ableger der Schweizer Sterbehilfeorganisation „Dignitas“ mit Sitz in Hannover. „Dignitas“ bietet wie auch „EXIT“ organisierte Suizidhilfe in der Schweiz an. Anders als „EXIT“ bietet „Dignitas“ ihre Dienste auch für Personen mit Wohnsitz im Ausland an. Bis 2012 betraf das rund $90 \%$ aller Suizidhilfen von „Dignitas“. 50 \% der Suizidhilfen wurden von Personen aus Deutschland in Anspruch genommen, in Gesamtzahlen ausgedrückt waren das 748 von insgesamt 1496 Freitodbegleitungen seit 1998. ${ }^{1}$ In Deutschland selbst tritt vor allem der vom ehemaligen Hamburger Innensenator Kusch gegründete „Sterbehilfe Deutschland e.V.“ in Erscheinung, der zusammen mit dem 2012 gegründeten Schweizer Ableger „Verein StHD“ seit 2010 insgesamt 77 Suizidbegleitungen vorgenommen hat. ${ }^{2}$

Die Politik zeigte sich alarmiert und reagierte auf diese Entwicklung seit 2006 mit der Forderung nach Strafbarkeit der Tätigkeit von Sterbehilfeorganisationen. Ein erster Entwurf der Länder Saarland, Thüringen und Hessen sah die Strafbarkeit der geschäftsmäßigen Vermittlung von Gelegenheiten zur Selbsttötung vor, ${ }^{3}$ wurde aber mangels einer Mehrheit im Bundesrat zurückgestellt. ${ }^{4}$ Ein Entwurf des Landes Rheinland-Pfalz hatte die Strafbarkeit der Werbung für Suizidhilfe zum Inhalt, ${ }^{5}$ fand aber ebenfalls keine

1 Vgl. Statistik „Freitodbegleitungen nach Jahr und Wohnsitz“ unter http://www.dignitas.ch/ images/stories/pdf/statistik-ftb-jahr-wohnsitz-1998-2012.pdf (zuletzt aufgerufen am 12.03.2012).

2 Pressemitteilung vom 1. Januar 2013 unter http://www.sterbehilfedeutschland.de/sbgl/files/ PDF/2013-01-01_PE.pdf (zuletzt aufgerufen am 12.03.2012).

3 BR-Drs. 230/06 vom 27.03.2006.

4 BR-Drs. 436/1/08 vom 04.07.2008.

5 BR-Drs. 149/10 vom 23.03.2010. 
Mehrheit im Bundesrat. ${ }^{6}$ Aktuell steht der Entwurf der Bundesregierung vom 22.10.2012 ${ }^{7}$ zur Schaffung eines neuen $\$ 217$ StGB mit folgendem Wortlaut zur Diskussion:

„\} 2 1 7 \text { Gewerbsmäßige Förderung der Selbsttötung }

(1) Wer absichtlich und gewerbsmäßig einem anderen die Gelegenheit zur Selbsttötung gewährt, verschafft oder vermittelt, wird mit Freiheitsstrafe bis zu drei Jabren oder mit Geldstrafe bestraft.

(2) Ein nicht gewerbsmäßig handelnder Teilnehmer ist straffrei, wenn der in Absatz 1 genannte andere sein Angehöriger oder eine andere ibm nabestehende Person ist."

Der Gesetzesentwurf ist vorerst gescheitert, freilich nicht aufgrund der besseren Einsicht, dass eine entsprechende Regelung ahistorisch, undogmatisch, verfassungswidrig und ethisch wie rechtspolitisch verfehlt wäre, sondern ganz im Gegenteil, weil konservative Kräfte innerhalb der CDU/CSU das Verbot jeglicher organisierter Sterbehilfe fordern. ${ }^{8}$

\section{Straflosigkeit von Suizid und Suizidhilfe}

\section{Suizid}

Schon in der Antike wurde der Suizid überwiegend nicht als Straftat angesehen, teils genoss er sogar den Status als natürliches Recht jedes freien Menschen; erst seit Aufkommen der christlichen Theologie und im Kirchenrecht wurde der Suizid als Unrecht gewertet, das aber nur Kirchenbußen zur Folge hatte. ${ }^{9}$ Rechtlich ist der Suizid in Deutschland schon Jahrhunderte toleriert worden. Bereits die Peinliche Gerichtsordnung Karls V. aus dem Jahr 1532 (Carolina) behandelt den Suizid nicht als Straftat. ${ }^{10}$ Dennoch wurde der Suizid auch noch bis über die Zeit der Aufklärung hinweg teilweise als straffrei, aber rechtswidrig angesehen. ${ }^{11}$

Das StGB kennt ebenso keine Strafbarkeit des Suizids bzw. Suizidversuchs. Dies findet seinen normativen Grund in der Verfassung. Die Straflosigkeit ist Ausdruck der verfassungsrechtlich gewährten Autonomie des Einzelnen. Die überholte Gegenposition ${ }^{12}$ würde eine Lebenspflicht begründen, die es nicht gibt ${ }^{13}$ und schon deshalb abzulehnen wäre, weil sie sich als Verkürzung und nicht als Verstärkung der grundrechtlichen Frei-

6 Vgl. BR-Plenarprotokoll 901, $441 \mathrm{C}$ vom 12.10.2012.

7 BT-Drs. 17/11126.

8 DÄBl. 2013, 110 (4), A 112; vgl. auch http://www.rp-online.de/politik/deutschland/unionwill-gesetz-zur-sterbehilfe-verschaerfen-1.3113671 (zuletzt abgerufen am 12.03.2013).

9 Vgl. den historischen Rückblick bei Dreier JZ 2007, $317 \mathrm{f}$.

10 Jakobs 1998, 5.

11 Jakobs 1998, $5 \mathrm{ff}$.

12 In diesem Sinne BGHSt (GrS) 6, 141, 153: niemand dürfe selbstherrlich über sein Leben verfügen. So erneut Lüttig ZRP 2008, 57, 58.

13 Bottke 1982, 49 f.; Dreier JZ 2007, 317, 319 m.w.N.

NK 25. Jg. 2/2013 
heitsgewährleistung darstellen würde. ${ }^{14}$ Somit ist der Ausschluss der Strafbarkeit von Suizid und Suizidversuch sogar verfassungsrechtlich geboten.

Umstritten ist die Frage, ob aus der Verfassung auch ein Recht auf einen freiverantwortlichen Suizid folgt. Ausdrücklich ist es nicht verbürgt. Das BVerfG hat zum Recht auf Suizid als Ausdruck eines selbstbestimmten Todes nicht Stellung genommen. Dennoch ist ein solches Abwehrrecht anzunehmen, wollte man sich nicht in Widerspruch zur Ausweitung der Autonomie des Einzelnen auch an dessen Lebensende setzen. Die Selbstbestimmung hat in den letzten Jahren eine beachtliche Aufwertung erfahren: einerseits durch die Rechtsprechung, insbesondere in der Entscheidung im Fall Putz aus dem Jahr 2010,15 in welcher der BGH das Recht auf den selbstbestimmten Tod im Falle des Behandlungsabbruchs hervorhebt und bei diesem auch aktive Tötungshandlungen akzeptiert. ${ }^{16}$ Andererseits durch den Gesetzgeber, der durch die Vorschriften des Patientenverfügungsgesetzes aus dem Jahr $2009^{17}$ die Beachtung des Patientenwillens verbindlich festlegt, und zwar gem. $\$ 1901$ a Abs. 3 BGB unabhängig von Art und Stadium einer Erkrankung, also unter ausdrücklicher Ablehnung einer Strömung, die mit einer sog. Reichweitenbeschränkung nur eine eingeschränkte Selbstbestimmung gewähren wollte. ${ }^{18}$ Gilt dies aber für den Fall des Behandlungsabbruchs oder -verzichts, kann für den ernstlichen und wohldurchdachten freiverantwortlichen Willen zum Suizid nichts anderes gelten. Das Selbstbestimmungsrecht hat sich dann gegen die staatliche Schutzpflicht durchzusetzen; denn ein Schutz gegen sich selbst würde im Falle des freiverantwortlichen Suizids in einen Paternalismus zurückführen, der mit dem Verständnis unserer freiheitlichen Grundordnung nicht vereinbar wäre.

Die dogmatische Herleitung des Rechts auf Suizid ist dagegen zweitrangig und ohne praktische Relevanz, mag sie aus dem Recht auf Leben als negative Freiheit (Art. 2 Abs. 2 GG), aus der allgemeinen Handlungsfreiheit (Art. 2 Abs. 1 GG) ${ }^{19}$ oder aus dem allgemeinen Persönlichkeitsrecht (Art. 2 Abs. 1 i.V.m. 1 Abs. 1 GG) hergeleitet werden. Stets greift ein Gesetzesvorbehalt. ${ }^{20}$ Dennoch erscheint das allgemeine Persönlichkeitsrecht als Rechtsquelle insofern vorzugswürdig, als bei der Frage des Rechts auf Suizid nicht die lebensbeendende Handlung als solche im Vordergrund steht, sondern die Ausübung des Selbstbestimmungsrechts für einen als würdevoll empfundenen, eigenen Tod.

14 Hillgruber 1992, 82.

15 BGHSt 55, $191 \mathrm{ff}$.

16 Rosenau 2011, 557; Wolfslast/Weinrich StV 2011, 286, 287; Dölling ZIS 2011, 345, 347; Rissingvan Saan ZIS 2011, 544, 547; Lipp FamRZ 2010, 1555, 1556; Gaede NJW 2010, 2925, 2927; Bartsch 2011, 23; Joerden 2011, 596 f.; vgl. auch Uhlig/Joerden Ad Legendum 2011, 369, 376, die die Sterbehilfe mittels Unterlassens wie durch Tun gemeinsam unter den Begriff indirekte Sterbehilfe fassen wollen, was wegen des belegten Begriffs der indirekten Sterbehilfe freilich nicht viel Klarheit brächte.

17 PatVG vom 29.07.2009, BGBl. I, 2286 f.: $\$ \int S 1901$ a ff. BGB.

18 Entwurf Bosbach u.a., BT Drs. 16/11360.

19 di Fabio, in: Maunz/Dürig (Begr.), GG, Loseblatt. 39. Lfg. 2001, Art. 2 Rn. 50; Bernat ÖJZ 2002, 92, 95.

20 Lindner NJW 2013, 136; vgl. auch Hufen NJW 2001, 849, 851. 
Der Europäische Gerichtshof für Menschenrechte (EGMR) verortet in der Entscheidung zum Fall Diane Pretty aus dem Jahr $2002^{21}$ das Recht auf Selbstbestimmung beim Sterben grds. in Art. 8 Abs. 1 EMRK, also dem Recht auf Achtung des Privatlebens. Ohne ein Recht auf Suizid ausdrücklich anzuerkennen ${ }^{22}$ geht er aber bzgl. der Hinderung eines Suizids von einem Eingriff in die Rechte des einzelnen aus; denn sonst hätte er nicht mehr Art. 14 EMRK im Hinblick auf eine Diskriminierung bzgl. der in der EMRK gewährten Rechte (hier Art. 8 Abs. 1 EMRK) prüfen können. ${ }^{23}$ Das wurde in einer jüngsten Entscheidung dadurch bestätigt, dass der EGMR sogar dem Ehemann einer suizidwilligen Frau attestierte, ein Eingriff in dessen Recht auf Selbstbestimmung nach Art. 8 EMRK habe vorgelegen, und dass der EGMR die deutschen Gerichte verurteilte, erneut in der Sache über die Zuteilung von Barbituraten zu entscheiden. ${ }^{24} \mathrm{Da}$ die Entscheidungen des EGMR aufgrund der gebotenen völkerrechtsfreundlichen Auslegung auch bei der Auslegung der Grundrechte nach dem GG zu beachten sind, ${ }^{25}$ auch wenn sie formal nur inter partes wirken (Art. 46 Abs. 1 EMRK), ist die weiterhin durch die Judikative des BGH wabernde Vorstellung vom Suizid als Unrecht ${ }^{26}$ somit heute nicht mehr haltbar.

\section{Suizidhilfe}

Der allgemeinen Strafrechtsdogmatik folgend kommt wegen der Akzessorietät der Teilnahme, die stets eine vorsätzlich begangene rechtswidrige Haupttat voraussetzt (vgl. $\mathbb{S} \$ 26,27$ StGB), eine Bestrafung wegen Beihilfe zum Suizid mangels Haupttat schon aus dogmatischen Gründen nicht in Betracht. ${ }^{27}$ Eine Pönalisierung der Beihilfe ist folglich im Grundsatz systemwidrig und daher abzulehnen. ${ }^{28}$ Das hat auch der Deutsche Juristentag 2006 in Stuttgart mit großer Mehrheit so gesehen und sich ausdrücklich gegen

21 Pretty vs. The United Kingdom, Application no. 2346/02, unter http://hudoc.echr.coe.int/ sites/eng/pages/search.aspx?i=001-60448 (zuletzt abgerufen am 12.03.2013); nichtamtliche deutsche Übersetzung: NJW 2002, $2851 \mathrm{ff}$.

22 Vgl. EGMR NJW 2002, 2851, 2854 (Rn. 67).

23 Faßbender Jura 2004, 115, 119.

24 EGMR, Urteil vom 19.7.2012, Koch ./. Deutschland, FamRZ 2012, 1465.

25 BVerfG NJW 2004, 3407, 3411; 2012, 3357, 3360.

26 So unter Berufung auf das „Sittengesetz“ BGHSt (GrS) 6, 147, 153; auch noch BGHSt 46, 279, 285.

$27 \mathrm{Zu}$ Recht ist die Gegenansicht von Bringewat ZStW 87 (1975), 623, 649 ohne Gefolgschaft geblieben.

28 Bottke R \& P 1993, 174, 181; a.A. Dölling, 129 f.; Tolmein ZfL 2013, 16, 18 unter Hinweis auf entsprechende Regeln im europäischen Ausland, die aber ihrerseits systemwidrig und alles andere als Ausdruck eines liberalen Strafrechts sind.

NK 25. Jg. 2/2013 
entsprechende Vorschläge ausgesprochen: „Die Poenalisierung der Teilnahme am Suizid ist - den Regeln der allgemeinen Strafrechtsdogmatik folgend - abzulehnen. “29

Fraglich ist, ob auch von Verfassungs wegen eine Strafbarkeit der Suizidbeihilfe generell ausgeschlossen wäre. Dagegen spricht zunächst, dass das Recht auf Suizid nur ein Abwehr-, nicht aber ein Leistungsrecht gewährt und damit die Hilfe zum Suizid anders bewertet werden könnte als der Suizid per se. ${ }^{30}$ Dennoch ist gesetzgeberische Zurückhaltung geboten, weil das Grundrecht auf Suizid nach der Bestimmung des Art. 19 Abs. 2 GG in seinem Wesensgehalt Bestand haben muss - insbesondere in Fällen, in denen der Suizidwillige auf die fremde Hilfe angewiesen ist (wie z.B. im Fall Diane Pretty). Wird eine Strafbarkeit des Suizidgehilfen unter bestimmten Voraussetzungen geregelt, können darüber hinaus eigene Grundrechte des Gehilfen betroffen sein, etwa die Berufsfreiheit von Ärzten gem. Art. 12 Abs. 1 GG im Falle einer Regelung zur ärztlichen Suizidhilfe. ${ }^{31}$ Es kann außerdem ein mittelbarer Eingriff in das Recht des Suizidwilligen vorliegen, der dann zu bejahen wäre, wenn die Folgen den Suizidwilligen besonders intensiv treffen würden. ${ }^{32}$ Freilich könnte auch der mittelbare Grundrechtseingriff durch ein Gesetz gerechtfertigt werden, das einen legitimen Zweck verfolgt und auch im übrigen dem Grundsatz der Verhältnismäßigkeit entspricht. Ob das der Fall ist, wird sogleich unter III. erörtert werden.

Die früher vom BGH vorgenommene Umgehung der Straflosigkeit der Suizidhilfe durch Begründung einer täterschaftlichen Unterlassensstrafbarkeit des hinzutretenden, in Unterstützung des Suizidwunschs untätig bleibenden Garanten ${ }^{33}$ - zuletzt im Fall Wittig $^{34}$ - ist jedenfalls im Hinblick auf die verfassungsmäßig garantierte Achtung des Selbstbestimmungsrechts auch beim freiverantwortlichen Suizid nunmehr als überholt anzusehen, weil mit dem freiverantwortlichen Suizid der Suizident sein Selbstbestimmungsrecht authentisch ausübt und insofern den Gehilfen von dessen Garantenpflichten entbindet. ${ }^{35}$ Dieses Ergebnis entspricht auch der gesetzgeberischen Wertung des $\ 1901 \mathrm{a}$ Abs. 2, Abs. 3 BGB. ${ }^{36}$ Entsprechend scheidet eine Strafbarkeit des Nichtgaranten wegen unterlassener Hilfeleistung gem. $\$ 323 \mathrm{c}$ StGB aus, da es beim freiverantwortlichen Suizid

29 Dieser Beschluss wurde mit dem Stimmenverhältnis 51:34:24 angenommen, Ständige Deputation des Deutschen Juristentages, Verhandlungen des Sechsundsechzigsten Deutschen Juristentages, Band II/2, 2006, N 204 u. N 207. Freilich hatte sich die Abteilung unmittelbar zuvor auch dafür ausgesprochen, die Förderung einer Selbsttötung aus Selbstsucht oder bei Ausbeutung einer $Z$ wangslage unter Strafe zu stellen, a.a.O. Beide Beschlüsse stehen erkennbar perplex gegeneinander, was zugleich ein bezeichnendes Licht auf die Weisheit des Deutschen Juristentages wirft.

30 Vgl. Kempf JR 2013, 11, 13.

31 Lindner NJW 2013, 136, 137; vgl. auch VG Berlin MedR 2013, 58 ff.; ablehnend Lorenz MedR $2010,823,828$.

32 Vgl. BVerwG NJW 1992, 2496, 2499.

33 Dölling 121.

34 BGHSt 32, 367 ff.; im konkreten Fall gelangte der BGH nur wegen Zumutbarkeitserwägungen nicht zu einer Strafbarkeit.

35 So auch schon OLG München NJW 1987, 2940, 2943.

36 Ausführlich Kutzer ZRP 2012, 135, $137 \mathrm{f}$. 
bereits an einem „Unglücksfall“ fehlt ${ }^{37}$ jedenfalls ein rettender Eingriff nicht erforderlich ist. $^{38}$

\section{Konkrete Einwände gegen den Regierungsentwurf}

Bezüglich des Regierungsentwurfs zur Strafbarkeit der gewerbsmäßigen Suizidförderung bestehen nach den o.g. Maßstäben erhebliche Bedenken rechtlicher, aber auch rechtspolitischer Art.

\section{Rechtliche Einwände}

Voraussetzung einer verfassungskonformen Strafnorm ist, dass diese dem Schutz anderer oder der Allgemeinheit dient; nur dann kann sie dem Verhältnismäßigkeitsgrundsatz entsprechen und die drohende Freiheitsentziehung rechtfertigen, die einen Eingriff in die persönliche Freiheit nach Art. 2 Abs. 2 Satz 2 GG darstellt. ${ }^{39}$ Schon hinsichtlich des geschützten Rechtsguts des $\$ 217$ StGB-E bestehen Zweifel. Denn wer sein Recht auf Suizid verwirklicht, muss sich dabei auch helfen lassen können, insbesondere in Fällen, in denen der Suizident sogar auf Hilfe angewiesen ist. Das Leben als Individualrechtsgut des freiverantwortlich handelnden Suizidenten kann daher nicht geschütztes Rechtsgut sein. ${ }^{40}$ Daran ändert auch die Voraussetzung des gewerbsmäßigen Handelns des Gehilfen nichts; denn gewerbliche Tätigkeiten, auch solche in Vereinigungen, sind ebenfalls grundrechtlich geschützt, so dass aus der Verknüpfung mehrerer grundrechtlich geschützter Verhaltensweisen keine verbotene gemacht werden kann. ${ }^{41}$ Überhaupt erscheint es systematisch verfehlt, mit rein strafschärfenden Merkmalen wie der Gewerbsmäßigkeit Strafbarkeiten zu konstituieren. ${ }^{42}$

Sofern der Gesetzgeber dagegen mit dem Entwurf einer abstrakten Gefahr für das Leben potentieller Suizidenten begegnen möchte, die in ihrem Suizidentschluss keineswegs gefestigt sind und sich aufgrund der Tätigkeit von Sterbehilfeorganisationen daher kurzentschlossen oder unter gefühltem Druck zu einem Suizid hinreißen lassen könnten ${ }^{43}$ würde sich grds. der Lebensschutz, der hohen Verfassungswert genießt, als legitimes Ziel der Regelung erweisen. Allerdings ist aufgrund der Gesetzesbegründung keineswegs das Bestehen, nicht einmal die Möglichkeit einer abstrakten Gefahr dargelegt. Zwar existiert ein gesetzgeberischer Beurteilungsspielraum bei der Einschätzung der (abstrakten) Gefahr, doch muss auch diese in den Realien des wirklichen Lebens fußen und darf nicht ins Blaue hinein behauptet werden. Es ist daher verfassungsrechtlich un-

37 Schönke/Schröder/Sternberg-Lieben/Hecker, StGB, \323c Rn. 7; Duttge u.a. 2004, 65 f.

38 OLG München NJW 1987, 2940, 2945.

39 BVerfGE 120, 224, 239.

40 Das konzedieren auch die Verfasser des AE-StB, die gleichwohl die gewinnsüchtige Suizidbeihilfe unter Strafe gestellt sehen wollen, vgl. Schöch/Verrel u.a. GA 2005, 553, 582.

41 Saliger ZRP 2008, 199.

42 Feldmann GA 2012, 498, 507; Fischer $\$ 217-\mathrm{E}, \mathrm{Rn} .4$.

43 So BT-Drs. 17/11126, 9, $6 \mathrm{f}$.

NK 25. Jg. 2/2013 
genügend, wenn sich die Beurteilung lediglich auf einen pauschalen Vergleich mit Sterbehilfezahlen aus dem Ausland und vage Befürchtungen stützt. Die im Gesetzesentwurf genannte Zunahme des Angebots entgeltlicher Suizidhilfe ist ebenfalls kaum belegt und ohne Zusammenhang zur behaupteten abstrakten Gefahr dargestellt.

Die Suizidzahlen in Deutschland pendeln seit dem Jahr 2000 um die Marke von 10.000 Suiziden pro Jahr. Während 2007 mit 9.402 Suiziden ein Tiefststand erreicht war, steigen seitdem die Zahlen allmählich leicht an und lagen im Jahr 2011 bei 10.144 Suiziden. ${ }^{44}$ Während aber die Entwicklung der Suizidzahlen von 2007 bis 2010 leicht steigend war, sanken im selben Zeitraum die Zahlen der Inanspruchnahme von Suizidbegleitung durch "Dignitas". ${ }^{45}$ Von 2010 bis 2011 stiegen die Zahlen jeweils: die Suizidzahl in Deutschland erhöhte sich von 10.021 auf 10.144, die Inanspruchnahme von Suizidbegleitung durch „Dignitas“ von 29 auf 72. Betrachtet man jedoch die Suizidmethoden, ist allein die Zahl an Suiziden durch Erhängen, Strangulieren und Ersticken ${ }^{46}$ um mehr als 115 Suizide angestiegen, während etwa die Suizide durch Einnahme von Pharmaka, die gängigste Methode der Sterbehilfeorganisationen, im selben Zeitraum insgesamt leicht rückläufig war. ${ }^{47}$ Aus diesen Zahlen lässt sich daher keine Gefahr durch die Tätigkeit von Sterbehilfeorganisationen erahnen, geschweige denn herleiten. Auch die vielfach zu lesenden Befürchtungen, mit der begrenzten Freigabe der aktiven Sterbehilfe in den BeneluxStaaten werde es zu vielfachem Missbrauch und einem Dammbruch gegen das Leben kommen ${ }^{48}$ haben sich nicht bestätigt, wie eine jüngst publizierte Studie belegt. Weder seien Missbräuche in nennenswertem Ausmaße belegbar noch stiegen die Fallzahlen weiter an. Sie hätten sich vielmehr auf dem Niveau vor der gesetzlichen Regelung der aktiven Sterbehilfe in den Niederlanden stabilisiert. ${ }^{49}$ Besteht aber bereits keine begründbare abstrakte Gefahr, fehlt es am legitimen Zweck des Gesetzes und somit an der Verhältnismäßigkeit. Im übrigen geht der Regierungsentwurf ohne nähere Begründung davon aus, dass Sterbehilfeorganisationen, um Gewinn zu erzielen, besonders schnell zur Durchführung der Suizidbegleitung geneigt sind, anstelle den Suizidwilligen zu beraten und ihm ggf. von seinem Vorhaben abzuraten oder sein Anliegen - etwa wegen offensichtlich fehlender Freiverantwortlichkeit - zurückzuweisen. Unabhängig davon, dass die einschlägigen Organisationen auch die Beratungstätigkeit satzungsmäßig festgeschrieben haben ${ }^{50}$ und diese bei „EXIT“ auch belegbar erfolgt, dürfte, würde man ihnen

44 Statistisches Bundesamt, Zahlen unter http://de.statista.com/statistik/daten/studie/75844/um frage/selbstmorde-in-deutschland-seit-2000/ (zuletzt abgerufen am 12.03.2013).

45 S. Link in Fn. 1.

46 ICD-10, Diagnoseschlüssel X70 unter http://www.gbe-bund.de/oowa921-install/servlet/oo wa/aw92/dboowasys921.xwdevkit/xwd_init?gbe.isgbetol/xs_start_neu/\&p_aid=i\&p_aid= $3446431 \&$ nummer $=670 \&$ p_sprache $=D \&$ p_indsp $=-55024 \&$ p_aid $=80148366$ (zuletzt abgerufen am 12.03.2013).

47 ICD-10, Diagnoseschlüssel X60-X62, Link wie Fn. 35.

48 Oduncu MedR 2005, 437, 444, zugleich ders. 2007, 88.

49 Onwuteaka-Philipsen/Brinkman-Stoppelenburg u.a., The Lancet, online publiziert am 11.7.2012 unter http://dx.doi.org/10.1016/S0140-6736(12)61034-4, 7 f. (zuletzt abgerufen am 02.04.2013).

50 StHD: $\mathbb{} 2$ Abs. 2-4; „Dignitas“: Art. 2; „EXIT“: Art. 2 Abs. 2-3. 
finanzielles Interesse unterstellen, ihr Interesse an einem lebenden Beitragszahler größer sein als an einem verstorbenen Mitglied. Andererseits ist nahe liegend, dass gerade die Entgeltlichkeit von Leistungen durch Sterbehilfeorganisationen im Vergleich zu möglichen kostenlosen Angeboten eher dämpfende Wirkung auf die Mitgliederzahlen hat. ${ }^{51}$ Der Gesetzesentwurf schafft somit, ohne dass wenigstens die reale Möglichkeit einer abstrakten Gefahr aufgezeigt würde, eine Strafbarkeit für Tätigkeiten, die noch weit vor der eigentlichen Suizidhilfe liegen; in der Tat ist nach der Formulierung von $\mathbb{2} 217$ Abs. 1 StGB-E für die Begründung der Strafbarkeit nicht einmal ein Suizidversuch notwendig, die "Gelegenheit“ dazu reicht aus. Dies stellt eine bedenklich weite Vorverlagerung der Strafbarkeit dar, die letztlich bedeutet, dass der Entwurf nur auf gesellschaftlich partiell geäußerte Empörung politisch reagiert und bloße Moralvorstellungen zum Maßstab des Strafrechts erhoben werden. ${ }^{52}$ Das verstößt gegen das „ultima ratio“-Prinzip im Strafrecht, welches zuletzt das BVerfG in seiner Inzest-Entscheidung nochmals dem Bundesgesetzgeber als zu wahrende Grenze für Strafgesetze in das Stammbuch geschrieben hat. ${ }^{53}$

Eine Strafbarkeitslücke, welche durch $\$ 217$ StGB-E zu füllen wäre, ist ebenfalls nicht ersichtlich, weil im Falle der Beteiligung bzw. Förderung eines nicht freiverantwortlichen Suizids jedenfalls eine Strafbarkeit wegen fahrlässiger Tötung gem. $\$ 222 \mathrm{StGB}$, bei Vorsatz sogar Totschlag in mittelbarer Täterschaft gem. $\mathbb{S}$ 212, 25 Abs. 1, 2. Alt. StGB in Betracht kommt. Damit scheitert der Entwurf auch mangels Erforderlichkeit an den Vorgaben für verfassungsgemäßes Strafrecht. ${ }^{54}$ Dem Risiko der mangelnden Beweisbarkeit fehlender Freiverantwortlichkeit ist jedenfalls nicht durch das Strafrecht zu begegnen, ${ }^{55}$ zumindest kann es das scharfe Instrument des Strafrechts nicht legitimieren. Hier ist vielmehr an die Schaffung von Kontrollen und Verfahren zur Ermittlung der Freiverantwortlichkeit zu denken.

Aber selbst bei Unterstellung einer abstrakten Gefahr für das Leben unentschlossener suizidbereiter Personen wäre die Vorschrift des $\$ 217$ StGB-E ungeeignet, der abstrakten Gefahr zu begegnen. Mit dem Merkmal „Gewerbsmäßigkeit“ lässt sich das jedenfalls nicht erreichen. Diese ist als Absicht definiert, sich durch wiederholte Tatbegehung eine fortlaufende Einnahmequelle von gewisser Dauer und gewissem Umfang zu verschaffen. ${ }^{56} \mathrm{Z}$ war ließen sich auch die Mitgliedsbeiträge, mit denen sich die Sterbehilfeorganisationen finanzieren, als mittelbare Vermögensvorteile qualifizieren, doch müsste sich die Gewerbsmäßigkeit dann gerade auf die Förderung der Suizidgelegenheit beziehen, was jedenfalls bei einem nur auf Kostendeckung bedachten System zweifelhaft ist; denn es geht bei der Gewerbsmäßigkeit um Gewinnerzielung. Eindeutig ins Leere geht der Gesetzesentwurf jedenfalls im Falle des „Sterbehilfe Deutschland e.V.“, der als Reaktion

51 A.A. offenbar Freund/Timm GA 2012, 491, 492.

52 In diesem Sinne auch Saliger ZRP 2008, 199.

53 BVerfGE 120, 224, $239 \mathrm{f}$.

$54 \mathrm{Zu}$ dieser Voraussetzung s. ebenfalls BVerfGE 120, 224, 240.

55 So aber Freund/Timm GA 2012, 491, 492.

56 BT-Drs. 17/11126, 9.

NK 25. Jg. 2/2013 
auf das Gesetzesvorhaben Mitgliedsbeiträge im Falle der erfolgten Suizidbegleitung wieder auszahlt. ${ }^{57}$

Andererseits dürfte auch die nach der Begründung des Entwurfs vorgesehene Ausnahme für Ärzte in $\$ 217 \mathrm{Abs}$. $2 \mathrm{StGB}-\mathrm{E}$, so sinnvoll sie an sich ist, ins Leere gehen, weil nicht eindeutig ist, unter welchen Kriterien ein Arzt eine „nahestehende Person“ ist. Hier versäumt der Entwurf, klare Rechtssicherheit für Ärzte herzustellen. Im übrigen dürfte nicht auszuschließen sein, dass auch Ärzte gewerbsmäßig handeln. Zwar sehen die einschlägigen Gebührenordnungen nicht vor, dass die Suizidbegleitung als eigenständige Leistung abgerechnet wird. Da sich diese aber im ärztlichen Alltag gar nicht von den allgemeinen ärztlichen Leistungen und Beratungen, die im Zusammenhang mit den Entscheidungen am Lebensende stehen, trennen lassen, ergibt sich bereits durch die allgemeine ärztliche Vergütung ein gewerbsmäßiges Handeln dann auch einer daran anschließenden Suizidhilfe. ${ }^{58}$

\section{Ethische und rechtspolitische Einwände}

Rechtspolitisch ist zu bemängeln, dass $\ 217$ StGB-E durch die Kriminalisierung von Sterbehilfeorganisationen Suizidwillige in den Brutal-Suizid drängt, weil diesen jegliche professionelle Hilfe vorenthalten wird. Vom Entwurf und den Stimmen, die diesen unterstützen, geht das fatale Signal aus, dass sich zwar jeder Mensch in Deutschland das Leben nehmen darf, dass er sich dazu aber von der Brücke zu stürzen, vor den Zug zu werfen oder den Strick zu nehmen habe. Diese sich nachgerade aufdrängende Konsequenz erscheint alles andere als ethisch vertretbar und dürfte den eigentlichen Abschied von der Humanität bilden. Sie wird aber allenthalben verdrängt oder verschwiegen. Umso mehr verwundern kirchliche Debattenbeiträge, die die Strafbarkeit der Suizidbeihilfe propagieren. ${ }^{59}$

In die Phalanx der Fürsprecher der Strafbarkeit jeder Form der organisierten Sterbehilfe hat sich auch die Bundesärztekammer als verbandspolitische Vertretung der Ärzteschaft eingereiht. ${ }^{60}$ Entsprechend haben sich die Ärzte eine Suizidassistenz bereits standesrechtlich selbst verboten. Nachdem zunächst die (nicht verbindlichen) Grundsätze der Bundesärztekammer zur ärztlichen Sterbebegleitung im Januar 2011 vorsichtig dahingehend gelockert wurden, dass die Mitwirkung des Arztes bei der Selbsttötung „keine ärztliche Aufgabe" sei, ${ }^{61}$ so dass also jeder Arzt ausschließlich seinem Gewissen

57 Satzung StHD $\$ 5$ Abs. 4.

58 Das Strafbarkeitsrisiko sieht auch Schreiber NStZ 2006, 473, 478.

59 Stellungnahme des Kommissariats der deutschen Bischöfe vom 4.6.2012, unter http:// www.kath-buero.de/files/Kath_theme/Stellungnahmen/2012/2012Nov 10Stellungnahme \%20Regierungsentwurf\%20Suizidhilfe.pdf (zuletzt abgerufen am 02.04.2013); Stellungnahme der Evangelischen Kirche in Deutschland und des Diakonischen Werkes der Evangelischen Kirche in Deutschland e.V. vom 29.5.2012 m.w.N., unter http://www.ekd.de/download/Ge meinsame_Stellungnahme\%281\%29.pdf (zuletzt abgerufen am 02.04.2013).

$60 \mathrm{Vgl}$. deren Stellungnahme zum hier diskutierten Referentenentwurf, ZfL 2012, 59, 60; s. auch Hoppe/Hübner 2010, $6 \mathrm{f}$.

61 Präambel, Abs. 7, DÄ 2011, 108 (7), A 346. 
unterworfen sein sollte, wurde nur wenige Monate später auf dem 114. Ärztetag in Kiel - gegen die ausdrückliche Empfehlung und mit großer Mehrheit verabschiedete Forderung des Deutschen Juristentages 2006 in Stuttgart ${ }^{62}-\$ 16$ MBO-Ä in der neuen Fassung verabschiedet, nach welcher Ärzte keine Hilfe zur Selbsttötung leisten dürfen. ${ }^{63}$ Diese Orientierungsvorschrift ist in 9 von 17 Landesärztekammern ${ }^{64}$ umgesetzt worden, 2 Landesärztekammern ${ }^{65}$ haben die vorhergehende Regelung beibehalten, 3 Landesärztekammern ${ }^{66}$ haben noch keine Entscheidung getroffen, die übrigen 3 Kammern ${ }^{67}$ haben abweichende, liberalere Regelungen getroffen. In diesem „Flickenteppich“ von Regelungen spiegelt sich auch die Uneinigkeit der Ärzteschaft in der Frage des ärztlich assistierten Suizids wider, ${ }^{68}$ während die Bevölkerung stark mehrheitlich die ärztliche Suizidassistenz unterstützt. ${ }^{69}$ Es ist zu bedauern, dass sich die Ärzte wie zu Zeiten Hippokrates' wieder vom Sterben in der Person des Sterbewilligen abwenden und sich aus der Suizidhilfe zurückziehen. ${ }^{70}$ Denn gerade diese haben die nötige Empathie, pflegen zumeist eine persönliche Bindung zum Patienten und verfügen über das nötige fundierte medizinische Wissen,${ }^{71}$ das für einen würdevollen Suizid erforderlich ist. ${ }^{72}$

Auch Sterbehilfeorganisationen sind nicht per se zu verteufeln. Sie können durchaus für ein Klima einer verantwortlichen Sterbebegleitung unter Einbeziehung der Angehörigen sorgen, zumal Ärzte dazu momentan nicht bereit sind. Im Ergebnis kann diese Begleitung dazu führen, dass der Suizid willige von seinem Suizidwunsch Abstand nimmt oder sich bereitfindet, in ein Hospiz zu ziehen und dort zu sterben. ${ }^{73}$ Die Organisationen schildern glaubhaft, dass sie durch ihre Tätigkeit zahlreiche Suizidwillige vom Suizid abhalten. Jedenfalls würde $\$ 217$ StGB-E geradezu kontraproduktiv wirken und Suizid-

62 Ständige Deputation des Deutschen Juristentages, Verhandlungen des Sechsundsechzigsten Deutschen Juristentages, Band II/2, 2006, N 204 u. N 207: Stimmenverhältnis: 72:27:12, wobei auch hier der Generalvorbehalt in Fn. 29 in Bezug auf solche Beschlüsse zu beachten ist. Gegen die Position der Ärzteschaft s. auch Verrel 2006, C $115 \mathrm{f}$.

63 DÄBl. 2011, 108 (38), A 1980, A 1984. Dazu Wenker Ethik Med (2013) 25: 73 ff.; ablehnend Wiesing Ethik Med (2013) 25: $67 \mathrm{ff}$.

64 Brandenburg, Bremen, Hamburg, Mecklenburg-Vorpommern, Niedersachsen, Nordrhein, Sachsen, Thüringen und Westfalen-Lippe (dort mit der Modifizierung, dass Ärzte keine Suizidhilfe leisten „sollen“).

65 Rheinland-Pfalz, Schleswig-Holstein.

66 Berlin (Entscheidung auf Delegiertenversammlung am 26.09.2012 vertagt), Hessen und Saarland.

67 Baden-Württemberg, Bayern und Sachsen-Anhalt.

$68 \mathrm{Vgl}$. dazu Allensbach-Umfrage vom Juli 2010 unter http://www.bundesaerztekammer.de/ downloads/Sterbehilfe.pdf (zuletzt abgerufen am 12.03.2013); Der Spiegel 32/2012, S. 114.

69 Vgl. dazu Iso Public-Gallup Umfrage vom Oktober 2012 unter http://www.medizinal recht.org/fileadmin/Dateien_fuer_News/Meinungsumfrageergebnisse_Selbstbestimmung am_Lebensende.pdf (zuletzt abgerufen am 12.03.2013).

70 In diesem Sinne auch Kreß 2011, 40.

71 Lorenz MedR 2010, 823, 827.

72 Vgl. Hillenkamp 2012, 1052; Schreiber 2007, 622; ders. 2013, 493.

$73 \mathrm{Zu}$ entsprechenden Erfahrungen aus Oregon Kreß 2011, 39.

NK 25. Jg. 2/2013 
willige sich selbst überlassen. Rechtspolitisch sendet der Entwurf das falsche Signal aus und stößt daher mit Recht auf weitgehende Ablehnung in der Bevölkerung. ${ }^{74}$

\section{Ergebnis}

Die von der Bundesregierung vorgeschlagene Strafbarkeit der gewerbsmäßigen Förderung der Selbsttötung ist ahistorisch, undogmatisch, verfassungswidrig und ethisch wie rechtspolitisch verfehlt.

Suizidprävention kann nicht über das Strafrecht erfolgen. Gegen eine öffentlich-rechtliche Regulierung und Überwachung der Tätigkeit von Sterbehilfeorganisationen, die im Falle des „Dr. Roger Kusch Sterbehilfe e.V.“ bereits funktioniert hat, ${ }^{75}$ ist jedoch nichts einzuwenden. ${ }^{76}$

\section{Literatur}

Bartsch, M. (2011) Sterbehilfe und Strafrecht - eine Bestandsaufnahme, in: Hellmann/ Schröder (Hrsg.), Festschrift für Achenbach, 13.

Bottke, W. (1982) Suizid und Strafrecht, zugl. München, Habilitation 1982.

Dölling, D. (2010) Zur Strafbarkeit der Mitwirkung am Suizid, in: Bloy u.a. (Hrsg.), Gerechte Strafe und legitimes Strafrecht. Festschrift für Manfred Maiwald, Berlin, 119.

Duttge, G. u.a. (2004) Preis der Freiheit.

Hillenkamp, Th. (2012) Suizidbeihilfe im Nahfeld - Der strafrechtliche Hintergrund, in: Anderheiden/Eckart, Handbuch Sterben und Menschenwürde, Bd. 2, 1033.

Hillgruber, C. (1992) Der Schutz des Menschen vor sich selbst, Studien zum öffentlichen Recht und zur Verwaltungslehre, Bd. 48, zugl. Köln, Dissertation 1991.

Hoppe, J.-D./Hübner, M. (2010) Ärztlich assistierter Suizid - Tötung auf Verlangen, Schriftenreihe Medizin-Ethik-Recht, Bd. 13.

Jakobs, G. (1998) Tötung auf Verlangen, Euthanasie und Strafrechtssystem, Bayerische Akademie der Wissenschaften, Sitzungsberichte, Heft 2.

74 Vgl. Iso Public-Gallup Umfrage vom Oktober 2012 (s. Fn. 67): nur 16 \% der Bevölkerung unterstützen das Bestreben, die Tätigkeit von Sterbehilfeorganisationen unter Strafe zu stellen.

75 Vgl. VG Hamburg MedR 2009, $550 \mathrm{ff}$.

76 Schreiber NStZ 2006, 473, 478; gegenteilig aber ders. 2007, 625; Hilgendorf JRE 2007, 479, 493; Birkner ZRP 2006, 52, 53; Kempf JR 2013, 11, 14. 
Joerden, J. C. (2011) Die neue Rechtsprechung zur Sterbehilfe und der Knobe-Effekt, in: Heinrich u.a. (Hrsg.), Festschrift für Claus Roxin zum 80. Geburtstag, 593.

Kreß, H. (2011) Patientenverfügungen, assistierter Suizid und Präimplantationsdiagnostik.

Oduncu, F. S. (2007) In Würde sterben.

Rosenau, H. (2011) Die Neuausrichtung der passiven Sterbehilfe. Der Fall Putz im Urteil des BGH vom 25.6.2010 - 2 StR 454/09, in: Bernsmann/Fischer (Hrsg.), Festschrift für Rissing-van Saan, 547.

Schreiber, H.-L. (2007) Strafbarkeit des assistierten Suizids?, in: Pawlik/Zaczyk u.a. (Hrsg.), Festschrift für Jakobs, 615.

Schreiber, H.-L. (2013) Das Recht auf den eigenen Tod - Zur gesetzlichen Neuregelung der Sterbehilfe, in: ders., Schriften zur Rechtsphilosophie, zum Strafrecht und zum Medizin- und Biorecht, 489.

Verrel, T. (2006) Patientenautonomie und Strafrecht bei der Sterbebegleitung. Gutachten C zum 66. Deutschen Juristentag.

Prof. Dr. Henning Rosenau

Akad. Rat Igor Sorge

Universität Augsburg, Juristische Fakultät

Lebrstubl für Deutsches, Europäisches und Internationales Straf- und Strafprozessrecht, Medizin- und Biorecht

Universitätsstr. 24, 86159 Augsburg 